\title{
Emergency-Management Situational-Awareness Prototype (EMSAP)
}

\author{
Kevin Adams, Alexander Wassell, Marion G. Ceruti, Senior Member, IEEE, \\ Ernesto Castro, Sandi F. Lehan, and John W. Mitchell \\ Space and Naval Warfare Systems Center Pacific (SSC Pacific) \\ 53360 Hull Street, San Diego, CA 92152-5001, USA \\ Tel. 001 (619) 553-4068', FAX 001 (619) 553-3931 \\ kevin.adams@navy.mil,alexander.wassell@navy.mil,marion.ceruti@navy.mil, \\ ernesto.castro@navy.mil, sandi.lehan@navy.mil,john.w.mitchell@navy.mil,
}

\begin{abstract}
This paper describes the architecture and requirements of an integrated system that is needed to support command and control for the interoperability-capability focus area. The architecture is designed to enhance situational awareness during emergencies such as earthquakes, volcanic eruptions, wild fires, floods, tsunamis, mud slides, storms, tornadoes, hurricanes, extreme heat, extreme cold, massive disease outbreaks, wars, terrorist attacks, power outages, cyber-attacks on utility grids and civil unrest. A net-centric approach that emphasizes cognitive aspects, such as cognitiveinformation operations, in decision support ensures information superiority by networking sensors and human-factors monitoring. This enables decision makers to achieve shared awareness. Linking knowledgeable entities effectively leads to increased speed of command and a higher tempo of operations with a degree of self-synchronization. The resulting system will decrease cognitive overload and improve cognitive monitoring by providing a more systematic and less labor-intensive method to manage information from and for first responders at the local, tribal, state, and federal levels.
\end{abstract}

Keywords: Cognition, Command and Control $\left(C^{2}\right)$; Decision Support; Net-Centric Services; Integration; Situation Awareness ${ }^{\dagger}$

\section{Introduction}

An integrated system is needed to support command-and-control interoperability emergency management. The purpose of the architecture is to en-

\footnotetext{
$\dagger$ The authors thank the Department of Homeland Security (DHS), the Office of Naval Research, and SSC Pacific's Business-Development Board for financial support.
}

hance situational awareness during emergencies such as earthquakes, volcanic eruptions, wild fires, floods, tsunamis, mud slides, storms, tornadoes, hurricanes, extreme heat, extreme cold, massive disease outbreaks, wars, terrorist attacks, power outages, cyber-attacks on utility grids and civil unrest. The architecture is designed to function with a net-centric approach that emphasizes the cognitive aspects of decision support, and ensures information superiority and mission success by networking sensors and human-factors monitoring.

The interoperability of current decision-support command-and-control systems across multiple government agencies is, at best, ad hoc across the military and homeland-security domains [1]. The goal of the architecture described here is to address and correct these deficiencies. Several components for a national strategy need to be addressed to solve the problem with imperatives for implementation of an integrated system that is sustainable both in terms of the technology and life-cycle logistical support. The following imperatives [1], [14] serve as components of a functionally integrated, servicecentric [17] emergency-management $C^{2}$ system.

- National Incident Management System (NIMS) and National Response Framework (NRF) for first responders in governments and private agencies.

- Outreach centers for technology transfer, including $\mathrm{C}^{4}$ ISR systems, Chemical, Biological, Radiological, Nuclear (CBRNE) defense [2], [6]; and human factors.

- Science and Technology (S\&T) assistance for architecture, data management, Cognitive Information Operations (CIO) [5], acquisition, sustainment.

This paper describes an architectural approach to support the Departments of Defense (DoD) and DHS with a particular emphasis on CIO, thereby improving interoperability and providing additional capability to various users communities. 


\section{Background}

Many of the components specified in the architectural design of this integrated system already are deployed and in use for various defense and DHS purposes. Thus, the integration involves not only mature systems that continue to prove their utility at a relatively high level of technological readiness, but future components now under development.

CIO needs to be integrated into emergency management in a more explicit manner. Originally, $\mathrm{CIO}$ was envisioned as a systematic way to understand and predict adversarial actions in asymmetric- and irregular-warfare environments. However, in disaster-relief situations, depending on the cause of the disaster, a clearly defined adversary is not always evident. Those in adversarial roles include but are not limited to terrorists in the case of manmade disasters, and opportunists who exploit for personal gain the chaotic conditions present in the aftermath of any disaster. To apply CIO to emergency management, we must understand how the adversary thinks, as thoughts precede all actions [5]. More timely and accurate information sharing can improve CIO. Moreover, CIO and the timely reporting of CIO-related events can enhance the overall disaster-relief mission.

Efficient Disaster Management (DM) consists of more than just the use of interoperable $\mathrm{C}^{2}$ components. It includes but is not limited to support for $\mathrm{CIO}$ and the dissemination of CIO-related information throughout the area of operation. First responders in various levels of government collaborate with private industry and private-relief organizations to implement systems and hardware working toward the goal to improve substantially the user-focused disaster-management resources.

\section{Cognition and Emergency Management}

Several aspects of cognition are important for situational awareness in emergency-management and disaster-relief situations.

- Sharing of information acquired during CIO contributes to overall situation awareness.

$\circ$ Ingroup-outgroup bias detection through language analysis [5].

$\circ$ Deception regarding group bias, e.g. falsely appearing to be a member of a group, or false denial of group membership.

- Deception in general [10]

- Communication of cognitive states

○ Normal mental conditions

- Cognition under stress [13]

- Fatigue, lack of sleep, and exhaustion

- Noise and thermal extremes

- Task urgency and time pressures

- Hypervigilance, rapid attention shifting
- Feeling of isolation due to scarcity of human and material resources.

- Mental impairment due to environmental hazards, e.g. CBRNE-related factors.

- Information overload can result not only when too much data must be analyzed quickly, but also when these data are not self consistent and are inconsistent with other observations.

EMSAP can help share information in reporting the results of CIO. For example, natural disasters, such as earthquakes, volcanic eruptions, and floods, cause widespread destruction of infrastructure and result in massive evacuation of personnel. Under these conditions, looting historically has been a problem. CIO is likely to be conducted while protecting vital infrastructure and deterring looters. During such a CIO, deception detection could become important when an individual posing as a store owner, actually wants entry for the purpose of looting.

Stress channels the attention to reduce focus on peripheral information and tasks and increase focus on main tasks [13]. Although selective attention can improve under stress [7], in general, stress degrades judgment and decision making [13]. Therefore, a commander or coordinator may want more information on the relative stress levels of personnel. A feature of the proposed standard Common Decision-Exchange Protocol (CDEP) [15] designed to track uncertainty in the decision-making process can be modified to support tracking and reporting of the cognitive state of various participants and groups in relief efforts. If a commander or disastermanagement coordinator is aware of the cognitive state of first responders and other relief personnel throughout the extended area of operation, the coordinator can allocate resources and make other decisions more efficiently.

Cognitive overload in DM can be reduced, but not eliminated entirely, by the sustainment of integrated, interoperable, and enhanced $\mathrm{C}^{2}$ systems.

\section{EMSAP Sustainment}

Sustainment requirements include the following.

- Maintenance of components necessary for migration to a net-centric environment and integration of common services.

- Development \& demonstration of a $\mathrm{C}^{2}$ Systems test strategy residing at the City and State Emergency Operations Centers that operate in an unclassified environment, and implementation of a test bed associated with vendor and government products available.

- Promotion and support of Service-Oriented Architecture (SOA) [17].

- Support for emerging services and sustainment of hardware and software interfaces. 
- Configuration management of data, common- component software and hardware interfaces, and the sustainment of a DHS S\&T resource repository with linkage to existing user-specific repositories and artifacts.
- Tracking the changing requirements associated with each agency's use of components and related interfaces will require extensive coordination and translation. Interfacing software for each mission-oriented capability will require changes.

\section{C4ISR Requirements ENABLERS Execution}

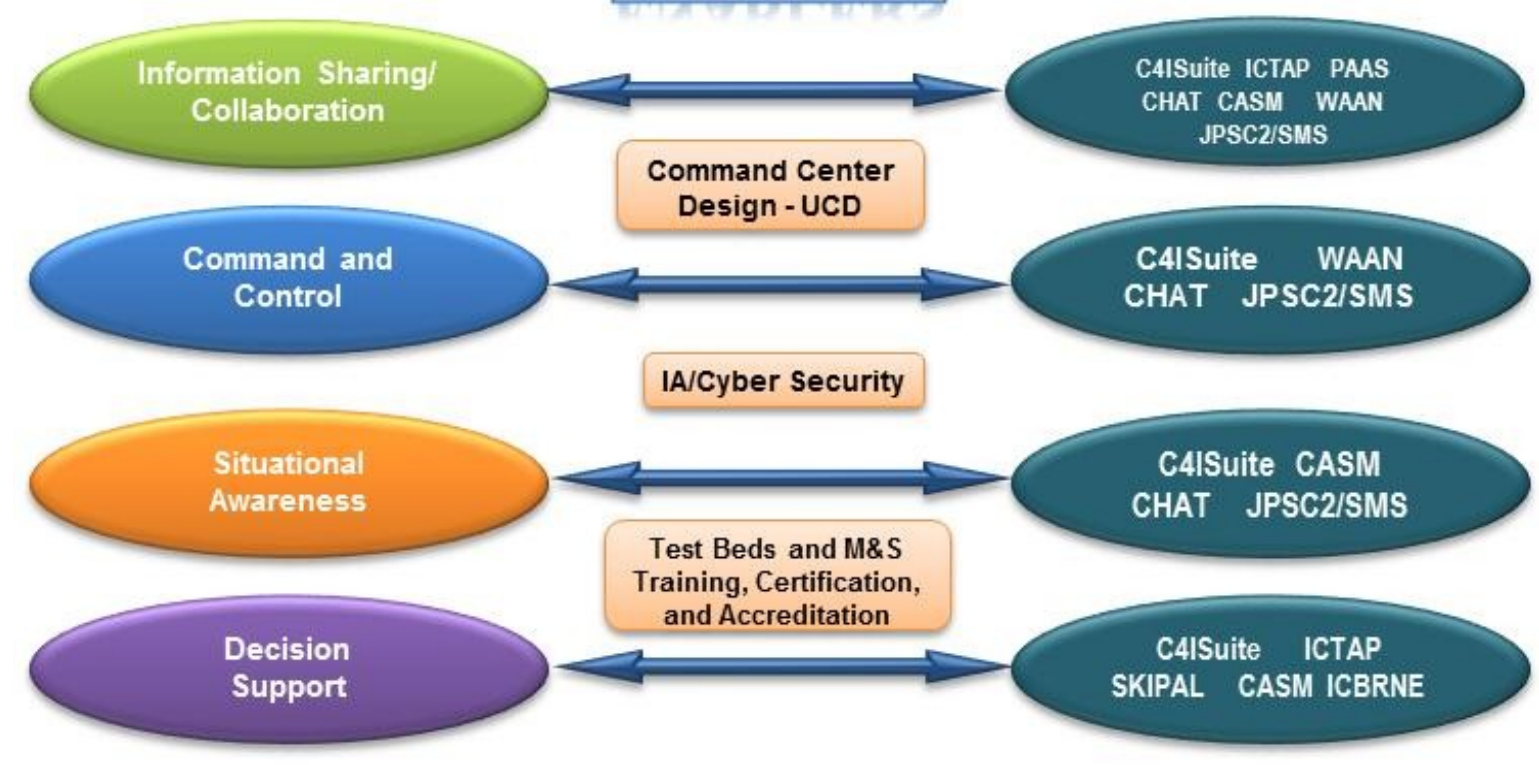

Fig. 1. Existing capabilities focused on emergency-management requirements [14]

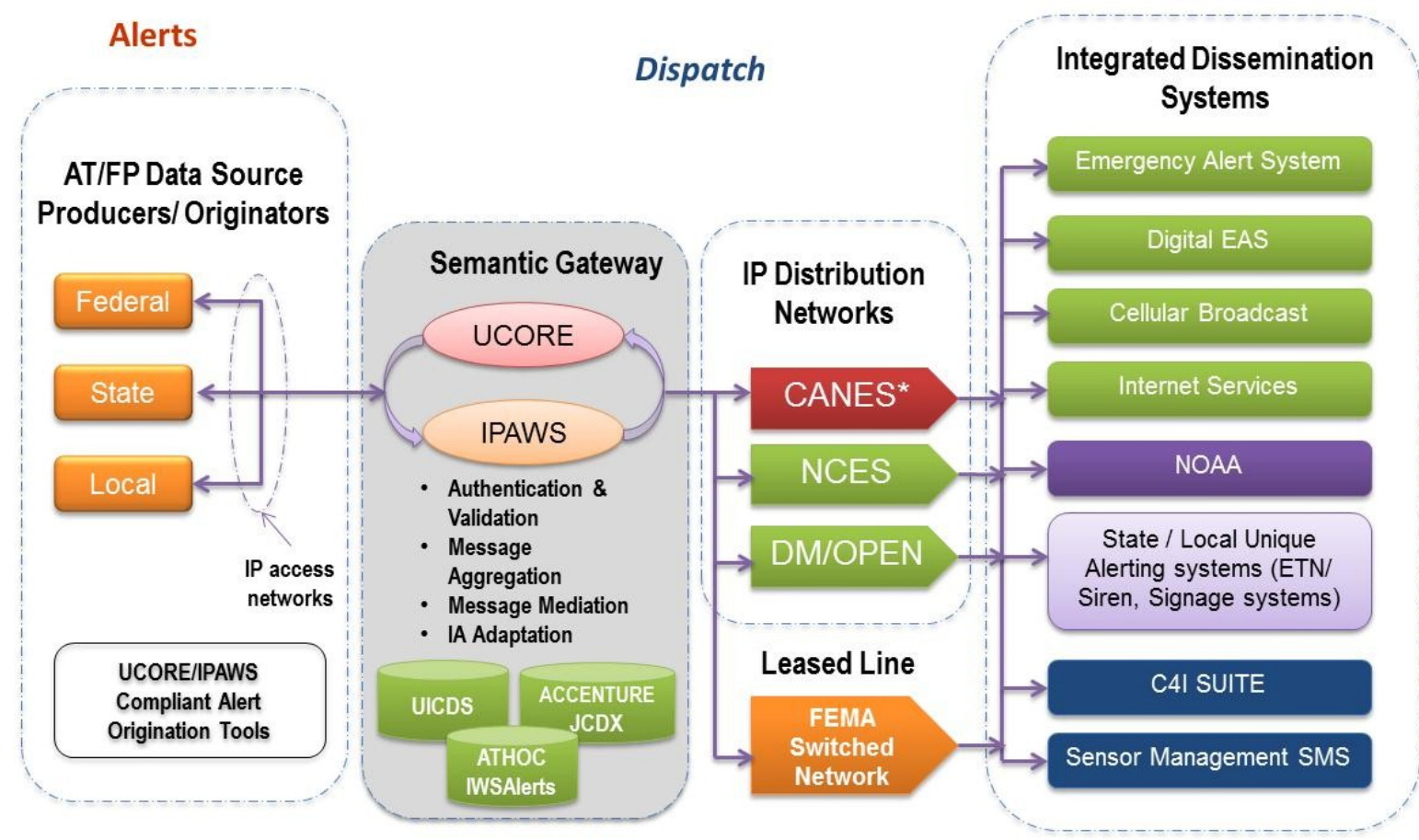

Fig. 2. Envisioned integrated-architectural framework of EMSAP using SOA-based technologies 


\section{Architecture for Cognitive Support}

Fig. 1 illustrates how to build relationships between requirements and existing state-of-the-art technology solutions for first responders by providing:

- Technology development \&transition

- Technology assistance \& outreach centers

- Information reuse

- Technology \& systems integration

- Modeling \& simulation center for first responders.

For example, $\mathrm{C}^{2}$ requirements include information sharing, collaboration, command, control, and situational awareness, as depicted in Fig. 1. Tools and technologies such as Chat [8] [11] support these and other military requirements. Chat can prove as valuable an asset for situation updates in future emergency management as it has for military communications.

Prior to chat technology, military personnel used radio as the primary means of tactical communication. This was very stressful and inefficient. The use of multiple radios for voice communications in a confined $\mathrm{C}^{2}$ space with many users produced an acoustical environment characterized by interrupted and/or garbled transmissions, static, interference, and an ever increasing volume of background noise as users would shout to be heard.

In contrast, chat communication has become the primary and often the singular means of communication in situation updates on intermittent and discontinuous networks, superseding radio communications with text-only descriptions [8]. Chat is popular because it improves the speed, clarity, and certainty of tactical communications, and because it provides a record for later reference and analysis that can be valuable from legal, strategic, and training perspectives. Chat supports the cross-domain operations and information sharing even without a common-operational picture [8].

The use of chat enhances the cognitive environment because it is quiet and enables users to focus on clear thinking rather than loud screaming. Therefore, chat users enjoy a significant reduction in stress, uncertainty, and fatigue that lead to cognitive failure and eventually, errors. Moreover, the use of visual icons can enhance the efficiency of chat, also increasing cognitive efficiency [8] [9].

Fig. 2 depicts the integrated-architectural framework of EMSAP using SOA-based technologies, including DoD and DHS component systems and services for first responders at all levels of government. The envisioned integrated architecture includes the next step toward semantic interoperability for federal, state, and local emergencyresponse personnel. Semantic interoperability clearly addresses and enhances the cognitive do- main. However, semantic-information integration has been the most challenging level of integration to achieve [3] [4] [16].

The architecture described in Fig. 2 features an integrated Universal Core (UCORE) semantic layer [12] and Integrated Public Alert and Warning System (IPAWS) as the heart of the semantic gateway to process alerts for emergency personnel from multiple echelons. The semantic gateway provides message aggregation and mediation. UCORE provides a general metadata foundation, fundamental components of an upper ontology, and an important step toward a comprehensive ontology of cognition for information sharing and understanding across multiple message domains [12].

\section{Future Directions}

An expanded discussion of the way forward can be found in [14]. Moreover, the following developments are necessary for a completely integrated $\mathrm{C}^{2}$ emergency-management system for first responders.

- Existing DHS S\&T programs need to be leveraged to provide funding to extend DoD open source $\mathrm{C}^{2}$ technology related to the following programs. The systems under development in these programs need to be integrated.

- Situational Awareness Geospatial Enterprise NORTHCOM Common Operational Picture

- Open architecture for city, state, and regional pilot program.

- San Diego local government and beyond, e.g. Fire department and water district.

- Future standards, protocols and systems need to be researched, developed, and integrated with existing systems to produce a comprehensive $C^{2}$ system [16]. For example, commanders, coordinators of first-responder teams and other emergency personnel will have the option to track states of uncertainty in decision making when a CDEP [15] is integrated into the integrated-architectural framework of EMSAP.

\section{Acknowledgments}

This paper is the work of U.S. Government employees performed in the course of employment and no copyright subsists therein. It is approved for public release with an unlimited distribution.

\section{References}

[1] K. Adams, S.F. Lehan, M.G. Ceruti, S.H. Rubin, and J. Waters, "Toward an Integrated System for Command and Control Net-Centric Web Services and 
Sustainment: Application to Homeland Security," Proceedings of the $11^{\text {th }}$ IEEE International Conference on Information Reuse \& Integration (IRI2010), pp. 347-377, Aug. 2010, Las Vegas, NV.

[2] M.G. Ceruti, "Chemical and Biological ThreatDetection Systems of the Future," Chem-Bio Defense Quarterly, Vol. 4 No. 2, pp. 18-21, 2007.

[3] M.G. Ceruti, and M.N. Kamel, "Semantic Heterogeneity in Database and Data Dictionary Integration for Command and Control Systems," Proceedings of the DOD Database Colloquium '94, pp. 65-89, Aug. 1994, San Diego CA.

[4] M.G. Ceruti, and M.N. Kamel, "Preprocessing and Integration of Data from Multiple Sources for Knowledge Discovery," International Journal on Artificial Intelligence Tools, (IJAIT), Vol. 8 No. 2, pp. 152-177, June 1999.

[5] M.G. Ceruti, S.C. McGirr, and J.L. Kaina, "Interaction of Language, Culture and Cognition in Group Dynamics for Understanding the Adversary," Proceedings of the National Symposium on Sensor \& Data Fusion, July 2010, Las Vegas, NV.

[6] M.G. Ceruti, R. Waters, P. Boss, R. Patel, S.H. Rubin, C. Datte, S. McGirr and T. Stark, "Defense CBRNE Threat-Detection System for Transportation Security," Proceedings of the IEEE International Conference on Technologies for Homeland Security (HST '08), pp. 519-524, May 2008.

[7] E. Chajut and D. Algom, "Selective Attention Improves Under Stress: Implications for Theories of Social Cognition," Journal of Personality and Social Psychology, Vol. 85, No. 2, pp. 231-248, 2003. http://shadowgov.1host.co.il/chajut\%20\&\%20algom $\% 20 \% 282003 \% 29$.pdf

[8] L.T. Duffy, E.W. Medina, S. Fugate, and G. Rogers, "Iconic and Text Chat: The Future of Tactical Text Chat," Proceedings of the First Annual Visual and Iconic Language Conference (VAIL 2007), 2-3 Aug. 2007, University of New Mexico, Albuquerque, $\mathrm{MN}$.

[9] S. Fugate, E.W. Medina, L.T. Duffy, D. Magsombol, O. Amezcua, G. Rogers and M.G. Ceruti, "NextGeneration Tactical-Situation-Assessment Technology (TSAT): Iconic Language," Proceedings of the First Annual Visual and Iconic Language Conference (VAIL 2007), 2-3 Aug. 2007, U. of New Mexico, Albuquerque, MN.

[10] D.R. Lambert, A Cognitive Model for Exposition of Human Deception and Counterdeception, NOSC TR 1076, Oct. 1987.

[11] E.W. Medina, S. Fugate, L. T. Duffy, D. Magsombol, O. Amezcua, G. Rogers and M.G. Ceruti, "Next-Generation Tactical-Situation-Assessment Technology (TSAT): Chat," Proceedings of the Seventh International Conference on Web Engineering (ICWE 2007), LNCS 4607 pp. 526-532, 16-20 July 2007, Como Italy.

[12] B. Smith, L. Vizenor, and J. Schoening, "Universal Core Semantic Layer," Proceedings of the 2009 Conference on Ontology for the Intelligence Community, Oct. 2009, George Mason U., Fairfax, VA.

[13] M.A. Staal, Stress, Cognition, and Human Performance: A Literature Review and Conceptual Framework, NASA Ames Research Center, Moffett Field, CA, NASA/TM-2004-212824, August 2004. http://humanfactors.arc.nasa.gov/web/library/publicat ions/publications.php

[14] J. Waters, K. Adams, S.F. Lehan, M.G. Ceruti, and S.H. Rubin, "Participation in IT-Standards Organizations Supports Multi-Agency First-Responder Command and Control System," Proceedings of the International Society for Computers and their Applications $23^{\text {th }}$ Intl. Conf. Computer Applications in Industry \& Engineering (CAINE 2010), Nov. 2010, Las Vegas, NV.

[15] J. Waters, M.G. Ceruti, R. Patel, and J. Eitelberg, "Decision-Acquisition System Based on a Common Decision-Exchange Protocol," Proceedings of the $15^{\text {th }}$ International Command \& Control Research \& Technology Symposium, (ICCRTS), June 2010, Santa Monica, CA.

[16] J. Waters, B.J. Powers and M.G. Ceruti, "Global Interoperability Using Semantics, Standards, Science \& Technology (GIS3T)," Journal of Computer Standards and Interfaces. Vol. 31 No. 6, pp. 1158-1166, 2009.

[17] D.R. Wilcox and M.G. Ceruti, "A Structured Service-Centric Approach for the Integration of Command and Control Components," Proceedings of the IEEE International Conference on Service Computing (SCC 2008), Vol. 2, pp. 5-12, July 2008.

\section{Appendix I. Acronym Glossary}

AT/FP-Anti-Terrorism/Force Protection

CANES-Consolidated Afloat Network \& Enterprise Services

CASM-Communication Assets Survey \& Mapping

DM/OPEN-Disaster Management message broker offering non-proprietary interfaces

EAS-Emergency Alert System

FEMA-Federal Emergency Management Agency

IA- Information Assurance

ICBRNE-Integrated Chemical Biological Radiological, Nuclear, Explosive (Program)

ICTAP-Integrated Communications Technical Assistance Program

IWS- Integrated Warfare Systems

JCDX- Joint Cross Domain Exchange

JPSC2-Joint Protection \& Surveillance Command $\&$ Control

M\&S-Modeling \& Simulation

NCES-Net-Centric Core Enterprise Services

NOAA-National Oceanic \& Atmospheric Administration

PAAS-Personnel Accountability \& Assessment System

SKIPAL-Strategic Knowledge Integration Personalized Assistant that Learns

SMS-Sensor-Management System

UICDS-Unified Incident Command \& DecisionSupport System

WAAN-Wide Area Alert Network 